\title{
Assessment of Student Learning of Operational Procedures in Electromagnetism
}

\author{
Yikun Han, Qiaoyi Liu², Feipeng $\mathrm{Pi}^{1, * *}$, Lei Bao ${ }^{2, *}$ \\ ${ }^{1}$ Guangzhou University, Guangzhou, Guangdong 510000, China \\ 2 Department of Physics, The Ohio State University, Columbus, Ohio 43220, United States \\ *Corresponding Author: pifeipeng@gzhu.edu.cn
}

(Received: 04/19/2021; Accepted: 06/14/2021; Published: 06/17/2021)

DOI: https://doi.org/10.37906/real.2021.3

\begin{abstract}
In the Chinese high school physics curriculum, three operational procedures are introduced in electromagnetism, namely right-hand grip rule, left-hand rule, and right-hand rule. This often led to confusion for student to differentiate these rules, and thus often focus on the surface characteristics of the problem or be misled by unrelated details. This study adopts the conceptual framework representation to model student understanding, and use it to guide the design of an assessment test on operational procedures in electromagnetism. The conceptual framework maps out the key concepts and their connections within a student's knowledge structure. In this study, an assessment tool of operational procedures in electromagnetism was developed and tested among Chinese high school students and college freshmen. Based on the testing results of the assessment, students have memory behaviors when using the right-hand grip rule, the left-hand rule and the right-hand rule, and students' understanding of electromagnetism can be summarized into three progression levels including novicelike, intermediate-mixed, and expert-like. Therefore, in order to help students develop integrated knowledge structures and deeper understanding, the cross product is an important central idea that needs to be emphasized in instruction.
\end{abstract}

Keywords: knowledge integration, operational procedures, electromagnetism

\section{Introduction}

In the 21st century, a new challenge has surged for students to adapt to the increase in complexity of the global society (Kelley \& Knowles, 2016). Therefore, it is essential for the modern STEM education to promote a deep understanding of science concepts (Koeninng, 2011), such that the students can apply them to solve complex problems in novel situations.

Founded upon the idea of establishing connections and coherence within students' knowledge structures, the knowledge integration framework (Linn, 2006) explains the two key difference between experts and novices are the connectedness and contextual dependence of their knowledge structures (Bao \& Koening, 2019; Hrdiman, Dufresne, \& Mestre, 1989; Laekin, Mcdermott, \& Simon, 1980; Dai, et al., 2019). Novices often have fragmented knowledge structures, and they can only apply them to a limited number of scenarios that they are familiar with through memorization (Bao \& Koening, 2019; Chi, Feltovich, \& Glaseer, 1981; Schoenfeld \& Herrmann, 1982). When faced with a novel situation, novices often rely on surface feature, and directly mapped them to the outcomes, with little conceptual understanding (Bao \& Koening, 2019). In contrast, experts usually have integrated knowledge structures, and they can apply them in different domains and solve problems with unfamiliar contexts. During problem solving, experts often 
first perform an initial qualitative analysis of a problem, to identify the central idea that is needed, before using the appropriate equation(s) for the solution of the problem (Bao \& Koening, 2019). When novices transition into experts, their approach to problem solving will also change. The mechanical method of relying on surface features to solve problems will transform to the systematic approach with consistent use of central ideas, while fragmented knowledge structure will transform to an integrated one (Bao \& Koening, 2019).

Several instruction methods have been used to promote the transition from novices to experts, such as peer instruction, clickers, studio learning, group discussions, learning by inquiry (Crouch \& Mazur, 2001; Keller, Finkelstein, Perkins, \& Pollock, 2007; Mcdermott, 1995), the transformative modeling framework (Shen, Liu, \& Chang, 2017), network (Kubsch, Nordine, Neumann, Fortus, \& Krajcik, 2018), and the conceptual framework. The conceptual framework is based on theories of conceptual change and knowledge integration, mainly used to model, evaluate and measure the level of knowledge integration, providing an operational tool for designing assessments and teaching interventions. The conceptual paths of novices and experts can be mapped in the conceptual framework. A key factor in evaluating students' level of knowledge integration is their ability to consistently use the central idea across a range of phenomena or contexts (Kubsch, Nordine, Neumann, Fortus, \& Krajcik, 2018; Nordine, Krajcik, \& Fortus, 2011). Experts arrange their knowledge structure in a hierarchy around a key central idea, where related ideas and conceptual components are linked to form conceptual pathways around the central idea, forming a fully integrated knowledge structure. They can solve problems across different contexts using this integrated knowledge structure by identifying and applying the central idea, a behavior best described as between relational and extended abstract in SOLO taxonomy. In contrast, novices often bypass the central idea and develop direct links amongst knowledge pieces, forming a fragmented knowledge structure. As a result, they often use mechanical problem solving methods such as pattern matching, where they match surface features of a problem to memorized algorithms and equations, a behavior best described as unistructural in SOLO taxonomy.

\section{Research Review}

In the field of electromagnetism, just like many other physics topics, students often hold many inaccurate ideas and erroneously interpret the central ideas (Bagno \& Eylon, 1997). For example, students often believe that magnetic poles as being charged and attract charged particles (Bagno \& Eylon, 1997), with the south pole of the magnetic field being positively charged. They also believe that the charged particles move in the direction of the magnetic lines of force in the magnetic field, and the Lorentz force they received points to electrode or the direction of the magnetic lines. When solving the problem of electromagnetic induction, they often fail to connect the concept of magnetic flux to Faraday's law, and believe that the induced magnetic field must be opposite to the original magnetic field (Greca \& Moreira, 1997; Albe \& Venturini, 2011; Maloney, O'Kuma, Hieggelke, \& Van, 2001). Finally, they often do not explicitly connect electromagnetic concepts with those in Newtonian mechanics (Planinic, 2006; Galili, 1995). In order to address these deficiencies, education researchers have been using advance technologies, such as Augmented Reality, to help student visualize physical scenarios and understand electromagnetic concepts (Kuncser, Kuncser, Maftei, \& Antohe, 2012; Abdusselam \& Karal, 2020; Saba, 1998). For example, researchers have used computers to design electromagnetic experiments exploring the phenomenon of electromagnetic induction (Onorato \& De Ambrosis, 2012), the magnitude and direction of the magnetic field force, and the connection between the force of a charged particle in a magnetic field and the force of a charged conductor in a magnetic field (Onotaro \& De Ambrosis, 2013).

In addition to the research on the teaching of fundamental concepts in electromagnetism mentioned above, there are a few studies on operational procedures as well, for instance, the right-hand rule. When the right-hand rule was created, physicists didn't fully understand the concept of cross product to 
determine the direction of the magnetic force, so the right-hand rule is a basic qualitative description of electromagnetic phenomena that helps us visualize and interpret electromagnetic law (Greenslade, 1980). After 1900, the right-hand rule was published in textbooks, and became an important operational procedure in electromagnetism. There are still various other operational procedures in high school physics textbooks in China and the United States recently. Other countries also use different types of rules. For example, when the direction of Ampere force, students in U.K. use Fleming's left-hand rule, those in Turkey uses right-hand rule, and those in Italy use the right-hand rule (Onotaro \& De Ambrosis, 2013).

Within the scope of electromagnetism taught in Chinese high school, there are three operational procedures, namely right-hand grip rule, left-hand rule, and right-hand rule. This often led to confusion for student to differentiate the right-hand rule and the left-hand rule, and thus will focus to the surface characteristics of the problem or be misled by unrelated details (Singh \& Rosengrant, 2003). Most recent studies on student difficulties focus on concepts in electromagnetism, and there is a lack of research on operational procedures such as the right-hand rule. Apart from this, China and other countries also create different operational concepts for different physical scenarios in textbooks. This could lead to different perspectives on electromagnetism for students from different counties, which requires integration from an objective lens.

The hypothesis of this study is that when students learn the right-hand grip rule, the left-hand rule, and the right-hand rule in Chinese high school physics textbook, most of them are memorized, which is easy to forget over time, and the knowledge structure becomes fragmented, and thus they lack deep conceptual understanding. Therefore, this study builds a conceptual framework from the perspective of knowledge integration based on the operational procedures in electromagnetism, and use it to design an assessment instrument that probes the connectedness of students' knowledge structures. The two main goals of this study are;

Establish a conceptual framework model for the topic of electromagnetism and apply the conceptual framework to map the reasoning pathways of novices and experts.

Assess whether there is memory behavior of students when they use right-hand grip rule, the left-hand rule, and the right-hand rule, and further explore the level of students' conceptual understanding of electromagnetism.

\section{Methods and Design}

\section{A. The Conceptual Framework of Electromagnetism Operational Procedures}

In this study, a conceptual framework was established to analyze student difficulties on electromagnetism operational procedures. Following the previous studies, when building a conceptual framework, the first step is to identify the central idea of the concept, which provides the core explanatory mechanisms or premises for establishing the causal relations underpinning the concept. In this case, cross product is chosen as the central idea for establishing the conceptual framework of electromagnetism operational procedures.

Cross product is an essential component of the mathematical language of physics (Knight, 1995). If there are two vectors, $\vec{a}$ and $\vec{b}$, with an angle $\theta$. The cross product results in another vector $\vec{c}=\vec{a} \times \vec{b}$, with magnitude $|\vec{c}|=|\vec{a}||\vec{b}| \sin \theta$, and direction perpendicular to the plane that vector $\vec{a}$ and $\vec{b}$ spans, which can be determined by Fleming's right hand rule. It is noting that Fleming's right-hand rule here is not the same as the right-hand rule taught in Chinese textbook. It refers to the case where the two vectors $\vec{a}$ and $\vec{b}$ are not perpendicular to each other, the four fingers point to the direction of vector $\vec{a}$, then find the angle with the smallest angle between the four fingers and vector $\vec{b}$, and bend the four fingers across this angle, then the direction of vector $\vec{c}$ is the direction pointed by the thumb. 
The right-hand grip rule is also known as the Ampere rule, which is used to determine the direction of the magnetic field around a current-carrying wire, described in the Chinese physics textbook as follows: hold the current-carrying wire with your right hand, the thumb points to the direction of the current, and the direction of the four curved fingers is the direction of the magnetic field lines. The right-hand grip rule can also determine the magnetic field from a circular current: when the four curved fingers of the right hand are bend in the same direction as the loop current, the direction pointed by the thumb is the direction of the magnetic field lines on the axis of the loop wire. The right-hand grip rule is a variant of the Fleming's right hand rule, therefore it also incorporates the concept of vector cross product.

The left-hand rule is used to determine the direction of Ampere force $\vec{F}_{\text {Ampere }}=I \vec{L} \times \vec{B}$ in Chinese textbook: let the magnetic field lines enter from the palm of left hand, the four fingers point in the direction of the current, the direction of the thumb is the same as the direction of the force on a current-carrying wire in a magnetic field. Due to the direction of $\vec{F}_{\text {Ampere }}$ is perpendicular to the plane spans by $\vec{L}$ and $\vec{B}$, so it is easy to use the right-hand grip rule to determine the direction of the Ampere force. That is, the four fingers of the right hand point in the direction of the current, grasp the direction of the magnetic field, and the thumb points in the direction of Ampere force.

The left-hand rule is also used to determine the direction of the Lorentz force $\vec{F}_{\text {Lorentz }}=q \vec{v} \times \vec{B}$ in Chinese textbook: let the magnetic field lines enter from the palm of the left hand, the four fingers point in the direction of the positive moving charge, the direction pointed by the thumb is the direction of the Lorentz force of the positive moving charge in the magnetic field. Similarly, the right-hand grip rule can be applied here, too.

When a part of the conductor is moving relatively through a magnetic field, the right-hand rule can be used to find the direction of the induced current caused by the electromotive force $\vec{\varepsilon}=l \vec{v} \times \vec{B}$ in the Chinese textbook: let the magnetic field lines enter from the palm of the right hand, the direction pointed by the thumb is the direction in which the wire is moving and the four fingers point in the direction of the induction current. Similarly, the right-hand grip rule can be applied here as well.

In its essence, the Ampere force and the induced current in the conductor are caused by the free charge moving due to the Lorentz force in the magnetic field. When a part of the conductor is moving through a magnetic field, the positive moving charge is subject to Lorentz force provided by the magnetic field and directional movement, then generating the induced current. Even though in actuality, the charge carriers in the conductor are electrons, and the direction of movement of electrons is opposite to that of positive charges, so the direction of induced current is opposite to the direction of electrons. As a result, in a section of the conductor without an external circuit, the positive and negative charges move to both ends of the conductor, and are held there by the Lorentz force, creating a potential difference. This fundamental connection of all these concepts from different topics of electromagnetism serves as the central idea of the conceptual framework.

In this study, the conceptual framework of operational procedures in electromagnetism, which contains the central idea and different reasoning pathways is shown in Figure 1. Solid arrows represent the experts' conceptual pathways, while the dashed lines represent the direct links between contextual features and the task outcomes that novices often make. The box at the top contains the central idea, which includes the cross product and involves two basic concepts of the cross product: the Biot-Savart law and the Lorentz force. From the cross product of the top box, the right-hand grip rule is derived. Experts usually use the right-hand grip rule to determine the direction of the magnetic field around a current-carrying wire and the magnetic field of a circular current, but can also replace the left-hand rule and the right-hand rule to determine the Ampere force, the Lorentz force, and the direction of the induced current in the conduct moving through the magnetic field, then directly complete the task goals. Since the experts have a full 
understand of the central idea, they can also relate other related concepts of electric field to those of magnetic fields. On the other hand, novices confuse the central idea of the three operational procedures, which are not unified. Their reasoning pathway starts from the right-hand grip rule, Ampere force, Lorentz force and induced electromotive force. They often treat these operational procedures as separate entities during problem solving, applying the right-hand grip rule to determine the direction of the magnetic field around a current-carrying wire and the magnetic field of a circular current, the left-hand rule to determine the direction of Ampere and Lorentz forces, the right-hand rule to determine the direction of the induced current in the conduct moving through the magnetic field. Through these three rules, the basic variables of electromagnetism are connected to achieve the task goals.

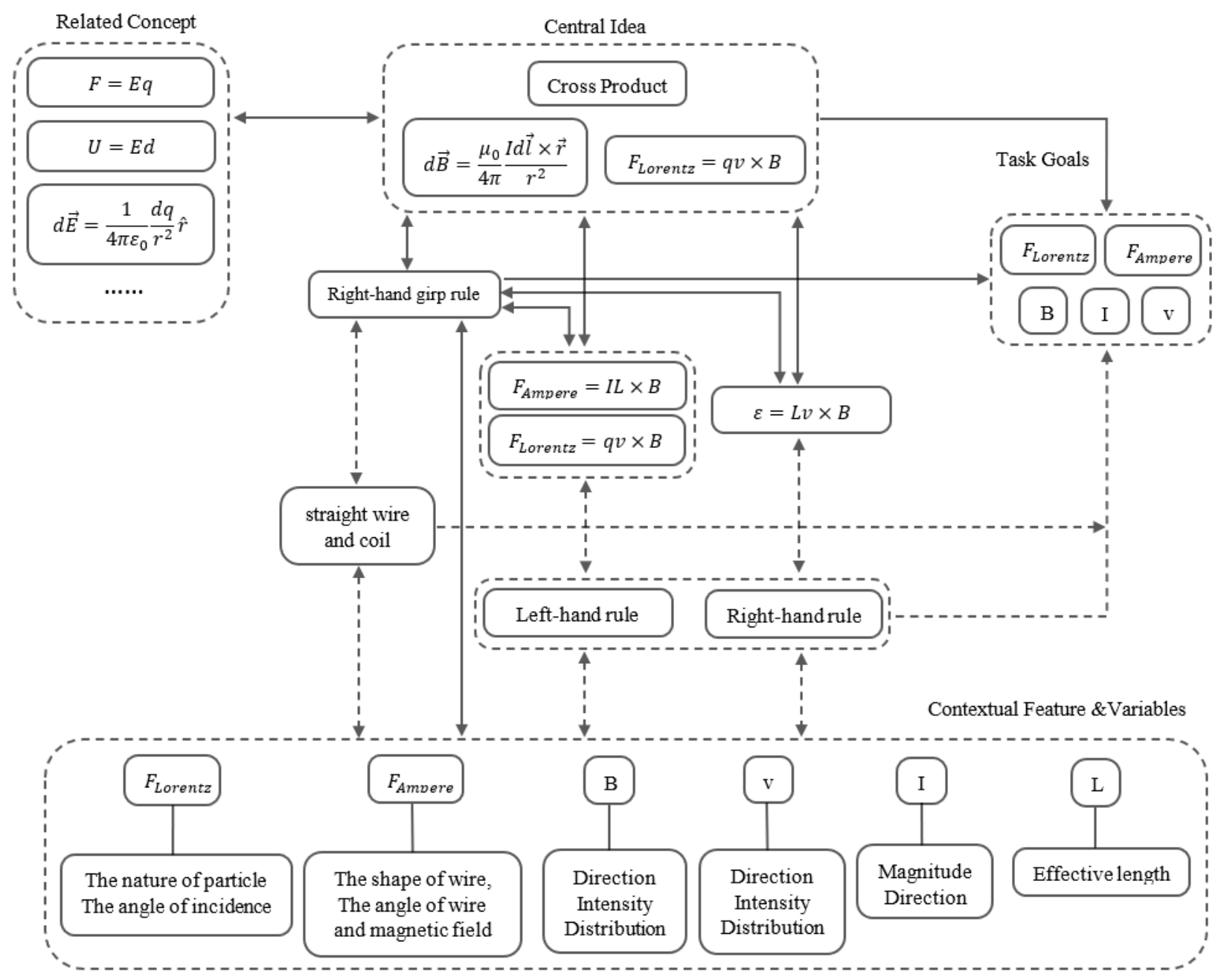

Figure 1: Conceptual Framework of the Operational Procedures in Electromagnetism

\section{B. Modeling Student Understanding using the Conceptual Framework}

To help understand how the conceptual framework manifests itself within students' knowledge structures, students' conceptual understanding and problem-solving behaviors can be summarized into three developmental levels, which are explained using the representative pathways of the conceptual framework: 
(1) Novice level: Students at this level have mostly fragmented knowledge structures, and are able to solve problems isomorphic to memorized example by directly applying the operational procedures. As a result, novices are often confused when faced with a new or slightly modified scenario that differs from those that they have seen. The novices' reasoning pathways are weak, direct links among the operational procedures, contextual feature and variables, and task goals. This type of behavior corresponds to the unistructural in SOLO taxonomy.

(2) Intermediate-mixed level: Students at this level can engage in a deeper level of reasoning compared to the novice students, but still tend to rely on memorized operational procedures to aid their problem solving. For example, when they determine the direction of the induced current in the conductor moving through the magnetic field, students in the intermediate-mixed level will consider whether it is more convenient to use Lenz's law. However, they fail to understand the central idea of this three rules, so they still lack a deep understanding of electromagnetism. If there is no obvious connection between variables and task goals, they will likely rely on memorized examples once again. This type of behavior corresponds to the multi-structural level in SOLO taxonomy.

(3) Expert level: Students at this level have developed a well-connected knowledge structure. This allows them to related contextual variables to the central idea, along with all the operational procedures, to form a comprehensive package of resources to address goal tasks. Therefore, they can solve any problem across different contexts.

Based on the difference in their problem-solving strategies, one can make inference on the connectedness of students' knowledge structures. In this study, the established conceptual framework and the description of different levels of students' understanding are used to guide the development of an assessment tool to evaluate the connectedness of students' knowledge strictures, and thus their conceptual understanding of the operational procedures in electromagnetism. Quantitative data were collected to analyze the general categories of students' knowledge structures. In addition, follow-up interviews were conducted to further examine students' thought process and reasoning pathways. These results will be used to validate the conceptual framework.

\section{Design of the Assessment}

Based on the operational conceptual framework of the operational procedures in electromagnetism, an assessment instrument was designed based on questions used in instruction and previous research on the related topics. The assessment contains 4 sections, each containing 4 multiple-choice questions, which evaluates students' conceptual understanding of the right-hand grip rule, the left-hand rule, the right-hand rule, and other related knowledge, as is shown in Table 1:

Table 1: Assessment items used in this study

\begin{tabular}{ccc}
\hline Concept Domain & Sections & Questions \\
\hline \multirow{3}{*}{ High school electromagnetic } & Right-hand grip rule & Q1, Q16, Q6, Q10 \\
knowledge & Left-hand rule & Q2, Q12, Q7, Q8 \\
& Right-hand rule & Q3, Q11, Q14, Q9 \\
& Other related knowledge & Q4, Q5, Q13, Q15 \\
\hline
\end{tabular}


In the right-hand grip rule section, students are asked to determine the polarity of a solenoid, the magnetic change of the electromagnet by adjusting the sliding rheostat; the distribution of the magnetic field around a circular loop current-carrying wire; or placing a small magnetic needle next to the solenoid and determine the twisting direction of the needle.

In the left-hand rule section, students are asked to determine the direction of the Ampere force and the Lorentz force in scenarios including: the force of the wire which is perpendicular or parallel to the magnetic field lines; the force of the closed current-carrying wireframe into the magnetic field; the trajectory of a positive charged particle which travels vertically into the magnetic field.

In the right-hand rule section, students are asked to determine the direction of induced current generated by a part of the conductor which is moving through a magnetic field; the direction of the induced current during the wireframe enters the magnetic field until exits the magnetic field.

The other related knowledge section contains topics relevant to physical quantities appear in the operational procedures of electromagnetism, such as the expression which is equivalent to the measurement of the strength of a magnetic field.

\section{Research Procedure}

The subjects of this study include 12th grade Chinese students taking a high school level physics course, along with the Chinese college freshmen who have not taken college-level physics course. The test was scheduled at the beginning of the 2020 spring semester. During a 50-min session, students were asked to complete the test online. A total of 531 students took the test, and the number of the valid questionnaires are 455 , with 244 high school students whose mean age was 17 years, 211 college students whose mean age was 20 years.

The primary purpose of this study is to assess whether there is memory behavior when students use the right-hand grip rule, the left-hand rule and the right-hand rule, evaluate their conceptual understanding of electromagnetism, examine the connectedness of their knowledge structures, and verify the conceptual framework of electromagnetism. To accomplish these research goals, some quantitative and qualitative methods were employed. Correlation analysis was conducted with both the high school students and the college students test data to identify the correlation coefficient related to the different context categories. The differences between the high school students and the college students across different question contexts were determined using t-test. The size of differences between the 12th grade students and the freshmen were measured with Cohen's f effect sizes. These quantitative methods can be used to evaluate memory behavior of the three rules, and also reveal the learning result of the operational procedures.

In addition to quantitative data, think-out-loud interviews were conducted with 19 Chinese students randomly selected from the same population pool but did not take the assessment. The interviews were conducted in a three-week time frame immediately after the completion of the quantitative assessment. As part of the interview, the students were asked to give their responses to each question while explaining their reasoning process. Each interview lasted about 30-40 minutes. All interviews were audiotaped and

transcribed, which were further analyzed to identify patterns of student reasoning for inference on students' knowledge structures.

\section{Analysis and Results}

A. Quantitative Study on Students' Knowledge Structure Development 
To explore the correlation between the right-hand grip rule, the left-hand rule, and the right-hand rule, the result of correlation analysis is showed in Table 2 and Table 3. The correlation coefficient among the right-hand grip rule, the left-hand rule, the right-hand rule of the high schools is between $0.2-0.34$, and the correlation coefficient among the three rules of the college students is between 0.44-0.51. Overall, there is a low correlation in the use of the three rules of between the high school students and college students, which means that students have not unified the operational procedures in electromagnetism. Compared to the high school students, college students did slightly worse when it comes to integrating these three rules.

Table 2: The correlation factors among the three rules for the high students

\begin{tabular}{lccc}
\hline & Right-hand grip rule & Left-hand rule & Right-hand rule \\
\hline Right-hand grip rule & 1 & 0.33 & 0.20 \\
Left-hand rule & 1 & 0.34 \\
Right-hand rule & & 1 \\
\hline
\end{tabular}

Table 3: The correlation factors among the three rules for the college students

\begin{tabular}{lccc}
\hline & Right-hand grip rule & Left-hand rule & Right-hand rule \\
\hline Right-hand grip rule & 1 & 0.51 & 0.44 \\
Left-hand rule & 1 & 0.47 \\
Right-hand rule & & 1 \\
\hline
\end{tabular}

The high school students and the college students' performances across different question sets are detailed in Figure 2 and listed in Table 4. The mean of four question sets of the college students is lower than that of the high school students. This is also confirmed by the t-test result, which shows no significant interaction between two student groups and the right-hand grip rule section $[t(453)=1.137, \mathrm{p}>0.05, \mathrm{~d}=$ 0.107]. The result shows significant differences in performance between the two student groups for the lefthand rule section [ $t(453)=3.594, p<0.05, d=0.334$ ], and the right-hand rule section [ $t(453)=2.438, p<0.05$, $\mathrm{d}=0.230]$. In addition, there is no significant interaction between two student groups and the other related knowledge section $[t(453)=1.260, p>0.05, d=0.118]$. 


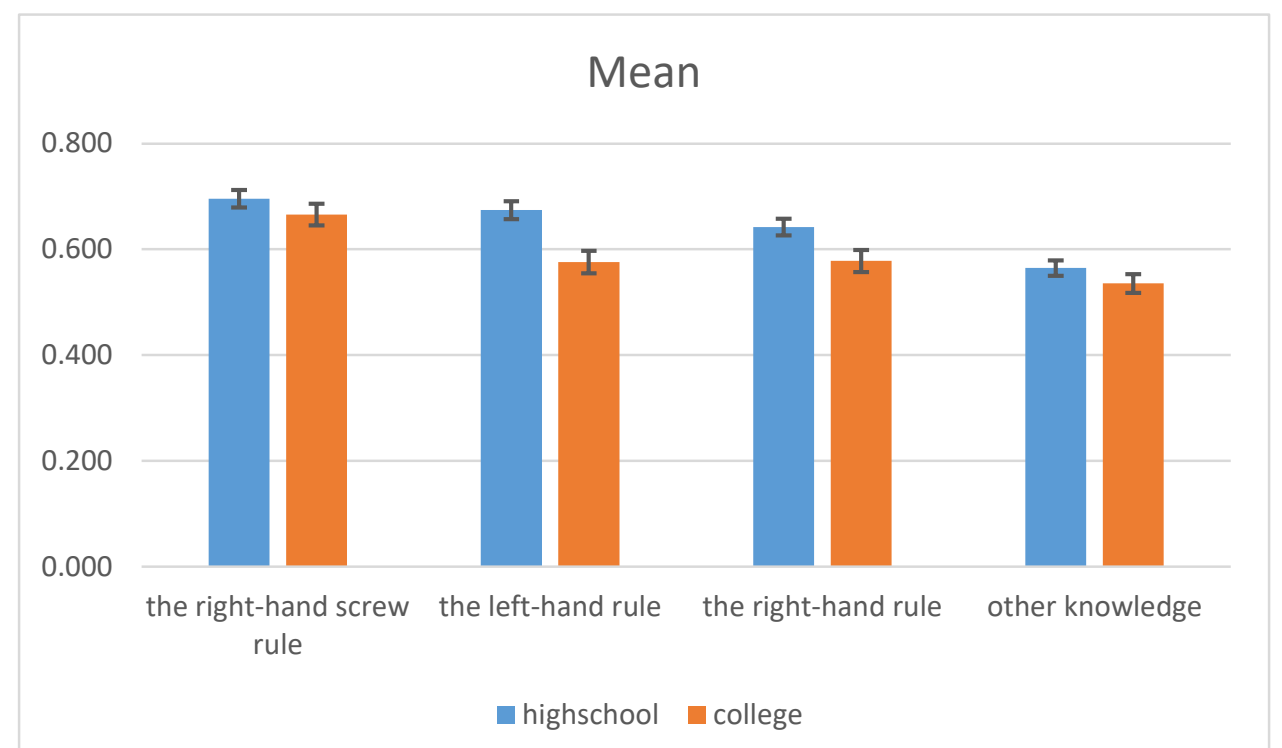

Figure 2: The mean of high school students and college students in four context categories. The error bars represent the standard errors.

Table 4: The statistical significance of differences in the four context categories

\begin{tabular}{cccccccc}
\hline \multicolumn{1}{c}{ Mean } & \multicolumn{2}{c}{ SE } & & & \\
& $\begin{array}{c}\text { High } \\
\text { school }\end{array}$ & college & $\begin{array}{c}\text { High } \\
\text { school }\end{array}$ & college & $\mathrm{t}$ & $\mathrm{p}$ & $\mathrm{d}$ \\
\hline $\begin{array}{c}\text { Right-hand } \\
\text { grip rule }\end{array}$ & 0.696 & 0.666 & 0.017 & 0.021 & 1.137 & 0.256 & 0.107 \\
$\begin{array}{c}\text { Left-hand } \\
\text { rule }\end{array}$ & 0.674 & 0.576 & 0.017 & 0.022 & 3.594 & $0.000^{*}$ & 0.334 \\
$\begin{array}{c}\text { Right-hand } \\
\text { rule }\end{array}$ & 0.642 & 0.578 & 0.016 & 0.021 & 2.438 & $0.014^{*}$ & 0.230 \\
$\begin{array}{c}\text { Other } \\
\text { knowledge }\end{array}$ & 0.565 & 0.536 & 0.015 & 0.018 & 1.260 & 0.208 & 0.118 \\
\hline
\end{tabular}

The results show no significant differences in performance between the high students and the college students in the right-hand grip rule section, indicating that the two groups of students are good at using the right-hand grip rule to determine the direction of the magnetic field around a current-carrying wire and a circular current. However, as the grade level gradually increases, there is a statistical significant difference in the use of the left-hand rule and the right-hand rule. It means that compared with high school students, college students are more likely to forget the content of the left-hand rule and the right-hand rule. Therefore, it is easier to make mistakes when determining the direction of the Ampere force, the Lorentz force and the induced current. 
In summary, a significant portion of high students and college students failed to obtain a deep conceptual understanding of the operational procedures in electromagnetism. A possible explanation for this result is that students mostly focus on how to memorize and apply the left-hand rule and the righthand rule without connecting them to the central idea, leaving them with fragmented knowledge structures.

\section{B. Qualitative Study on Students' Reasoning Process}

In order to identify students' possible reasoning pathways, 19 Chinese students were randomly selected from the same population pool to participate in think-out-loud interviews using the same assessment questions. As part of the interview, students were asked to solve the questions while explaining in detail how they got their answers. Based on their responses, three levels of students' understanding were identified and described below.

Novice level: students at this level solve problems by relying on memorizing the operational procedures with little reasoning. The contextual variable and features cue their memory of related operational procedures without meaningful connections to other related conceptual components and ideas. The knowledge structure is largely fragmented with only local connections that link contextual variables directly to equations. Students at this level can solve only a limited number of familiar problems, most of which they have encountered during their prior coursework. Interview excerpts from students who exhibit this type of problem-solving behavior are given below:

Student A: (response to question 11) "The teacher said, "the left hand is an electric motor because we use the left-hand rule to determine the Ampere force and the Lorentz force, the right hand is a generator because we use the right-hand rule to determine the induced current or the velocity of a part of the conductor is moving through a magnetic field."

Students B: (response to question 11) "I don't know how to solve it. I remember that the quantity in the question is expressed by hand rules, but it is not clear the context of the left-hand rule and the right-hand rule.

These students, like most typical novices, could only answer questions by matching contextual features and variables to memorized operational procedures. For instance, student A strictly followed the operational procedure in the textbook, and chose the appropriate rules based on the required physics quantities. It is showed that students at this level use different methods to deal with different physical scenarios, and they failed to identify the central idea of the three rules. For example, in question 7, when the wire rotates outside the paper, there must be a velocity perpendicular to the paper, when students know the direction of the velocity of the wire and the current, they can also get the direction of the magnetic field according to the right-hand rule. Students also failed to understand the basic reasoning for the currentcarrying coil moves because of the magnetic field force. It can be seen that students at this level lack a deep understanding of electromagnetism. Moreover, they can still make mistakes even if they have identified the correct operational procedure. For example, in question 13, the conductor in an open circuit should also experience the Ampere force when it moves in a magnetic field. However, students confuse the conditions for the Ampere force generation with the conditions for the induced current generation. This means that their knowledge framework is incomplete. For instance, student B still remembers the left-hand rule is related to the force of the magnetic field, and the right-hand rule is related to the induced electromotive force, but the specific content of these two hand rules couldn't be clearly described. They are also confused about the physical quantity represented by each finger, and mainly rely on habits to solve questions (such as question $3,11,14)$. When students explain what would happen when a part of the conductor moves through a magnetic field, they often believe that nothing would happen. This shows that students failed to understand the force of electric charges in a magnetic field is part of the central idea of electromagnetism, and thus cannot relate it to the other physical quantities. 
Intermediate-mixed level: students at this level can engage in a deeper level of reasoning based on the contextual variables compared to the novice students. However, they still tend to rely on memorized examples and equations to aid their problem solving. The knowledge structures of these students have more links with emerging network-like constructs that to some extent overlap with the experts' network of connections. Although there still memorize results for the left-hand rule and the right-hand rule, they make some inferences and judgments when dealing with special physical scenarios, and determine whether the operational procedure is applicable or not. However, students at this level still depend on the left-hand rule and the right-hand rule, while ignoring the cross product. Interview excerpts from students who exhibit this type of problem-solving behavior are given below:

Student C: (response to question 11) "When the conductor ab moves to the right, the number of magnetic lines entering the wireframe increases. According to the Lenz's law, in order to maintain the original state, a counterclockwise current with the magnetic lines facing out of the paper will be generated. I am not used to the right-hand rule because I need to try my best to figure out the physical quantities of every finger. And I can't apply the cross product to solve this problem.

Student D: (response to question 7) "The current ab moves out of the paper and receives a force out of the paper, the current cd moves into the paper and receives a force into the paper. If the direction of the magnetic field is vertically shown in option $C$, the current of the wire ab points from a to $b$. According to the right-hand rule, it can be seen that the force of the wire $a b$ is outward of the paper, and the force of the current cd is inward of the paper.

Students at the intermediate-mixed level are more flexible in choosing methods. For example, student $\mathrm{C}$ chose Lenz's law instead of the right-hand rule to determine the direction of the induced current of the wire c. He thought clearly about the physical quantities represented by the four fingers and the thumb and distinguished it from the left-hand rule before using the right-hand rule, so Lenz's law will be more convenient and intuitive. However, these students who rely on memorizing these rules are still prone to mistakes. In addition, students at this level often consider the options first before solving the problem, most prominently shown in the performance in question 7 . Students at the intermediate-mixed level are able to understand electromagnetism from the perspective of particles, but they still fail to use cross product to determine the direction of the magnetic field force and the induced current, which often leads them to incorrect answers.

Expert-like level: students at this level have the most developed knowledge structures. They are not limited to the conditions given, but often extend the known physical quantities to other implicit physical quantities, summarize the commonalities of different physical scenarios and choose one method to solve multiple physical problems. They use the right-hand grip rule along with the cross product instead of the left-hand rule and the right-hand rule separately to determine the direction of the Ampere force, the Lorentz force, and the induced current of a part of the conductor is moving through a magnetic field. Experts understand the force and the movement of a single charge in a magnetic field, and establish a wellconnected knowledge structure.

Student E: (response to question 6) "When the conductor AB slides to the right, according to the righthand grip rule, the four fingers point to the right and grasp the direction of the magnetic field. The direction of the magnetic field is perpendicular to the paper surface, the thumb points from $B$ to $A$, so the induced current direction is A-D-C-B-A.

Students at the expert-like level not only understand electromagnetism from the perspective of particles, they have also connected all the operational procedures with the cress product, achieving deep understanding of electromagnetism. 


\section{Conclusion}

In this study, a conceptual framework of the operational procedures in electromagnetism was developed, and was used to guide the development of the assessment instrument to probe students' knowledge structures in learning electromagnetic concepts. Based on the qualitative and quantitative analysis of the assessment outcomes, students have memory behaviors when using the right-hand grip rule, the left-hand rule and the right-hand rule, and students' understanding of electromagnetism can be summarized into three progression levels including novice-like, intermediate-mixed, and expert-like.

The novice-like students struggled with most of the questions, and their understanding appeared to be closely tied to surface features of the problems without deeper connections to the central idea. They seemed to have memorized aspects of the concepts in fragments, which appeared to be directly tied to the contextual features and variables. Consequently, they were unable to provide meaningful reasoning or explanations to their answers, and were only able to answer a few questions that could be easily matched to previously memorized examples.

The students at the intermediate-mixed level were able to answer most or all the electromagnetism questions, however, they had significant difficulties with questions involving the cross product. Their knowledge structures seemed to be locally connected among conceptual components and contextual variables, with few connections made to the central idea. As result, their problem-solving strategies were more flexible but failed to relate to the central idea.

The expert-like students were able to answer most or all of the questions by appropriately applying the cross product. They appear to have successfully developed deep conceptual understanding around the central idea with well-established connections among most key variables and relations, forming an integrated knowledge structure. Therefore, when solving problems, these students were able to focus on the central idea and use it to guide their problem-solving strategies without being tied to the surface features of the problem contexts.

The problem-solving behavior of students at different stages reveals the process of students' understanding from superficial to deep, from relying on scenes-driven memory and then gradually using the central idea to connect various concepts. This process reflects the ability of students to integrate knowledge fragments, and their knowledge structure has gradually evolved from fragmented to integrated.

In conclusion, this study extends previous work on the use of the conceptual framework into the topic of operational procedures in electromagnetism. The results show that instruction with a primary emphasis on the different hand rules in electromagnetism can leave students in the novice or transitional stage with fragmented knowledge structures. In order to help students develop integrated knowledge structures and deeper understanding, the cross product is an important central idea that needs to be emphasized in instruction. This study further demonstrates that the conceptual framework approach is effective in modeling and analyzing knowledge integration in learning physics, and can also provide valuable guidance on developing instructional methods that promote deep learning.

Conflicts of Interest: The authors declare no conflict of interest.

\section{References:}

Abdusselam, M., \& Karal, H. (2020). The effect of using augmented reality and sensing technology to teach magnetism in highs chool physics. Technology,Pedagogy and Education, 1-18. 
Albe, V., \& Venturini, P. (2011). Electromagnetic concepts in mathematical representation of physics. Journal of Science Education and Technology, 10(2), 197-203.

Bagno, E., \& Eylon, B. (1997). From problem solving to a knowledge structure: An example from the domain of electromagnetism. American Journal of Physcis, 65(8), 726-736.

Bao, L., \& Koening, K. (2019). Physics education research for 21st century learning. Disciplinary and Interdisciplinary Science Education Research, 1(1), 1-12.

Chi, M., Feltovich, P., \& Glaseer, R. (1981). Categorization and respersentation of physics problem by experts and novices. Cognitive Science, 5(2), 121-152.

Crouch, C., \& Mazur, E. (2001). Peer instruction: Ten years of experience and results. American journal of physics, 69(9), 970-977.

Dai, R., Fritchman, J., Liu, Q., Xiao, Y., Yu, H., \& Bao, L. (2019). Assessment of student understanding on light interference. Physical Review Physics Education Research, 15(2), 020134.

Galili, I. (1995). Mechanics background influences students' conceptions in electromagnetism. International journal of science educatio, 17(3), 371-387.

Greca, I., \& Moreira, M. (1997). The kinds of mental representations--models, propositions and images-used by college physics students regrading the concept of field. International Journal of Science Education, 19(6), 711-724.

Greenslade, J. (1980). Ancestors of the right-hand rule. The Physics Teacher, 18(9), 669-670.

Hrdiman, P., Dufresne, R., \& Mestre, P. (1989). The relation bewteen problem categorization and problem solving among experts and novices. Memory \& cognition, 17(5), 627-638.

Keller, C., Finkelstein, N., Perkins, K., \& Pollock, S. (2007). Research-nased Practices For Effective Clicker Use. AIP Conference Proceedings. American Institute of Physics, 951(1), 128-131.

Kelley, T., \& Knowles, J. (2016). A conceptual framework for integrated STEM education. International Journal of STEM Education, 3(1), 11.

Knight, R. D. (1995). The vector knowledge of beginning physics student. The Physcics Teacher, 33(2), 7477 .

Koeninng, J. (2011). Assessing 21st century skills:Summary of a workshop.

Kubsch, M., Nordine, J., Neumann, K., Fortus, D., \& Krajcik, J. (2018). Measuring Integrated KnowledgeA Network Analytical Approach. International Society of the Learning Sciences,Inc.[ISLS].

Kuncser, C., Kuncser, A., Maftei, G., \& Antohe, S. (2012). Computer assisted methods in teaching basics of magnetism at high school level. Romanian Peports in Physics, 64(4), 1119-1130.

Laekin, J., Mcdermott, J., \& Simon, D. (1980). Expert and novice performance in solving physics problem. Science, 208(4450), 1335-1342.

Linn, M. (2006). The knowledge integration perspective on learning and instruction.In R.K Sawyer(Ed). In The Cambridge handbook of the learning sciences (pp. 243-264). New York: Cambridge University Press.

Maloney, D., O'Kuma, T., Hieggelke, C., \& Van, H. (2001). Surveying students' conceptual knowledge of electricity and magnetism. American Journal of Physics, 69(S1), S12-S23. 
Mcdermott, L. (1995). Physics by Inquiry: An Introduction to Physics and the Physics Sciences. John Wiley \& Sons.

Nordine, J., Krajcik, J., \& Fortus, D. (2011). Trandforming energy instruction in middle school to support integrated understanding and future learning. Science Education, 95(4), 670-699.

Onorato, P., \& De Ambrosis, A. (2012). Magnetic damping:Integrating experimental and theoratical analysis. American journal of physics, 80(1), 27-35.

Onotaro, P., \& De Ambrosis, A. (2013). How can magnetic forces do work? Investigating the problem with students. Physics Education2013, 48(6), 766.

Planinic, M. (2006). Assessment of difficulties of some conceptual areas from electricity and magnetism using the Conceptual Survey of Electricity and Magnetism. American Journal of Physics, 74(12), 1143-1148.

Saba, M. (1998). Right-hand rule with magnet and monochrome monitor. The Physics Teacher, 36(3), 190190.

Schoenfeld, A. H., \& Herrmann, D. J. (1982). Problem perception and knowledge structure in expert and novice mathematical problem solvers. Journal of expermental Psychology:Learning.Memory, and Cognition, 8(5), 484.

Shen, J., Liu, O., \& Chang, H. (2017). Assessing students' deep conceptual understanding in physics sciences: An example on sinking and floating. International Journal of Science and Mathematics Education, 15(1), 57-70.

Singh, C., \& Rosengrant, D. (2003). Mulitiple-choice test of energy and momentum concepts. American Journal of Physics, 71(6), 607-617. 